\title{
BMJ Open Cluster-randomised controlled trial to assess the effectiveness and cost- effectiveness of an obesity prevention programme for Chinese primary school- aged children: the CHIRPY DRAGON study protocol
}

\author{
Bai Li, ${ }^{1}$ Wei Jia Liu, ${ }^{2}$ Peymane Adab, ${ }^{1}$ Miranda Pallan, ${ }^{1}$ Karla Hemming, \\ Emma Frew, ${ }^{1}$ Rong Lin, ${ }^{2}$ James Martin, ${ }^{1}$ Wei Liu, ${ }^{2}$ Kar Keung Cheng ${ }^{1}$
}

To cite: Li B, Liu WJ, Adab P, et al. Cluster-randomised controlled trial to assess the effectiveness and costeffectiveness of an obesity prevention programme for Chinese primary school-aged children: the CHIRPY DRAGON study protocol. BMJ Open 2017;7:e018415. doi:10.1136/ bmjopen-2017-018415

- Prepublication history and additional material for this paper are available online. To view these files, please visit the journal online (http://dx.doi. org/10.1136/bmjopen-2017018415).

Received 3 July 2017 Revised 9 October 2017 Accepted 18 0ctober 2017

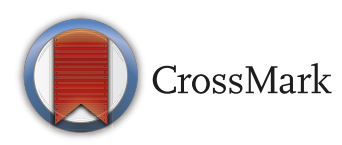

${ }^{1}$ Institute of Applied Health Research, College of Medical and Dental Sciences, University of Birmingham, Birmingham, UK ${ }^{2}$ Faculty of School Health, Guangzhou Centre for Disease Control and Prevention, Guangzhou, PR China

Correspondence to Professor Peymane Adab; p.adab@bham.ac.uk

\section{ABSTRACT}

Introduction Childhood obesity in China has increased more rapidly and over a shorter time period than in other countries. However, there is a paucity of rigorously developed and evaluated prevention interventions. We aim to evaluate the clinical and cost-effectiveness as well as the implementation process of a complex multicomponent intervention developed using the UK Medical Research Council (MRC) framework. This study provides one of the first examples of rigorous development and evaluation of a childhood obesity prevention programme in a non-western population using the MRC methods.

Methods and analysis A cluster-randomised controlled trial in 40 primary schools in Guangzhou, China, including children aged $6-7$ years at baseline. Schools will be randomly allocated to either the usual practice $(n=20)$ or intervention arm $(n=20)$. The 12-month intervention consists of four components targeting diet and physical activity behaviours in and outside school, with family involvement. The primary objective is to compare the difference in mean body mass index (BMI) $z$-score between the intervention and control arms at the end of the intervention (starting March/April 2017). A sample size of 1640 pupils recruited from 40 schools is sufficient to detect a difference of 0.17 units in the mean BMI z-score with a power of $80 \%$ (ICC $=0.01$. ICC, intraclass correlation coefficient) and a significance level of $5 \%$. Treatment effects will be tested using a mixed linear model in STATA adjusting for the child baseline BMI z-score and clustering by school. All analyses will be by intention to treat. Secondary analyses will additionally adjust for prespecified school-level and child-level covariates. The incremental cost-effectiveness ratio for the intervention versus usual practice will be 'cost per quality-adjusted life year (QALY)'. Cost per change in BMI z-score will also be assessed. A range of methods will be used to evaluate intervention implementation, mechanisms of impact and contextual factors.

Ethics and dissemination Ethical approval was obtained from the Life and Health Sciences Ethical Review Committee at the University of Birmingham and the Ethical

\section{Strengths and limitations of this study}

- This study provides one of the first examples of rigorous evaluation of a childhood obesity prevention programme in a non-western population using the methods set out by the UK Medical Research Council.

- We include an explicit process evaluation plan and incorporate behavioural change techniques in the design of the intervention.

- The sample size is large enough to detect a clinically meaningful effect and cost evaluation will be conducted from a societal perspective using validated tools.

- Contamination between the two arms cannot be avoided completely, although we anticipate the risk for contamination is small and have set out strategies to minimise such a risk.

- The intervention development process, trial design and evaluations of intervention process and costeffectiveness would inform future prevention research and practice in childhood obesity in China and beyond.

Committee of Guangzhou Centre for Disease Control and Prevention. The primary, secondary, process evaluation and economic evaluation results of the trial will be disseminated through relevant international peer-reviewed journals and conferences.

Trial registration number ISRCTN11867516; Pre-results.

\section{BACKGROUND}

Childhood overweight and obesity has become a major public health challenge worldwide. ${ }^{1}$ It is linked to serious health problems in both childhood ${ }^{2}$ and adulthood. ${ }^{3}$ In comparison with normal weight children, obese children are at greater 
risk of premature mortality. ${ }^{4}$ Obesogenic behaviours can persist from childhood to adulthood, ${ }^{5}$ and more than half of obese children are likely to become obese adults. ${ }^{6}$

In China, the prevalence of overweight and obesity among school-aged children has increased more rapidly and over a shorter time period than other countries, ${ }^{78}$ with the prevalence in some large cities approaching the level of developed countries. ${ }^{910}$ There is an urgent need to develop effective culturally appropriate prevention interventions to control the epidemic. However, there has been little research applying rigorous methods and established theoretical tools to develop and evaluate prevention programmes for Chinese children. The most recent Cochrane review of previous childhood obesity prevention trials showed that school-based interventions targeting children aged 6-12 years were effective in reducing adiposity. ${ }^{11}$ Nevertheless, several methodological flaws were identified, including poor reporting of process and implementation measures, lack of equity analyses in relation to sex and socioeconomic status, short-term follow-up and paucity of reports on costs and potentially harmful effects.

\section{Development of a childhood obesity prevention programme} Using the framework set out by the UK Medical Research Council (MRC) for complex intervention development and evaluation, ${ }^{12}{ }^{13}$ we developed an obesity prevention programme for Chinese children aged 6-7 years-the CHIRPY DRAGON study (CHInese pRimary school children PhYsical activity and DietaRy behAviour chanGes InterventiON). A number of different methodologies were employed and iteratively combined in the development process. ${ }^{14}$ The theoretical and modelling phases (2009-2012) included a mixed-methods study ${ }^{16}$ which integrated (1) focus groups and interviews with key stakeholders $(n=99)$ to explore perceived causes of childhood obesity and perceptions of effective and feasible intervention ideas as well as delivery preferences, ${ }^{1718}$ and (2) a cross-sectional study $(n=497)$ that identified several modifiable factors associated with child weight status and obesogenic behaviours. ${ }^{19}$ This resulted in six potential intervention targets. A further qualitative study (2012-2014, n=114) was conducted with a focus on contextual barriers and facilitators to implementing specific intervention components. It gathered key stakeholders' opinions on the identified potential interventions as well as three intervention components that had been included in a previous obesity prevention trial conducted in local primary schools but lacked implementation evaluation. We also reviewed international and Chinese literature on modifiable risk factors for obesity in Chinese children. ${ }^{20}$ Overall, the design of the intervention programme (intervention components and delivery strategies) was informed by the findings of these studies and incorporated social marketing principles ${ }^{21}$ and behaviour change techniques as defined by the Coventry, Aberdeen \& London
- Refined-Taxonomy. ${ }^{22}$ A feasibility/pilot study in three Chinese primary schools in 2015 helped to test and optimise the implementation and acceptability of the intervention programme, ready for evaluation in a definitive trial (CHIRPY DRAGON).

In this paper, we describe the study protocol for the definitive evaluation which is a cluster-randomised controlled trial.

\section{Trial aims and objectives}

The trial aims to assess the clinical and cost-effectiveness of the 12-month childhood obesity prevention intervention programme developed and refined through the previous phases, using usual practice in primary schools as the comparator. Intervention effects will be examined at two time points, immediately following the intervention completion and then 12 months later. The research objectives for the trial were: (1) to assess the effectiveness of the intervention compared with usual practice in preventing childhood overweight and obesity and the incremental cost-effectiveness of the intervention (primary outcomes); (2) to assess the effectiveness of the intervention on diet, physical activity and quality of life in children (secondary outcomes); (3) to assess whether any effects differed by sex, social class or baseline body mass index (BMI; subgroup analyses) and (4) to undertake process evaluation to assess the extent to which contextual and implementation parameters explain the observed trial outcomes.

\section{Trial design and overview}

The CHIRPY DRAGON study is a cluster-randomised controlled trial. Forty primary schools will be recruited from the city of Guangzhou, China. Randomisation will be at the level of school (cluster). Data will be collected at both the school level and from individual children and their parents. To test the effect of the intervention, a range of anthropometric and psychological data will be collected (detailed later) from children within participating schools. Baseline measures will be undertaken when the participating children are in the autumn term of year 1 . Schools will then be randomly allocated to either the usual practice or intervention arm. Schools in the intervention arm will be provided with a 12-month, multicomponent programme when they start the spring term of year 1 (detailed below). The programme includes physical activity and dietary components, targeting school and family environments and aims to facilitate the development and/or maintenance of a healthy weight. The first follow-up measures will be undertaken immediately after the intervention year when the children are in Year 2) and the second follow-up measures are undertaken 12 months later when the children are in Year 3). The study design and timelines are shown in figure 1.

The trial was prospectively registered with ISRCTN registry (ISRCTN11867516) on 19 August 2015. 


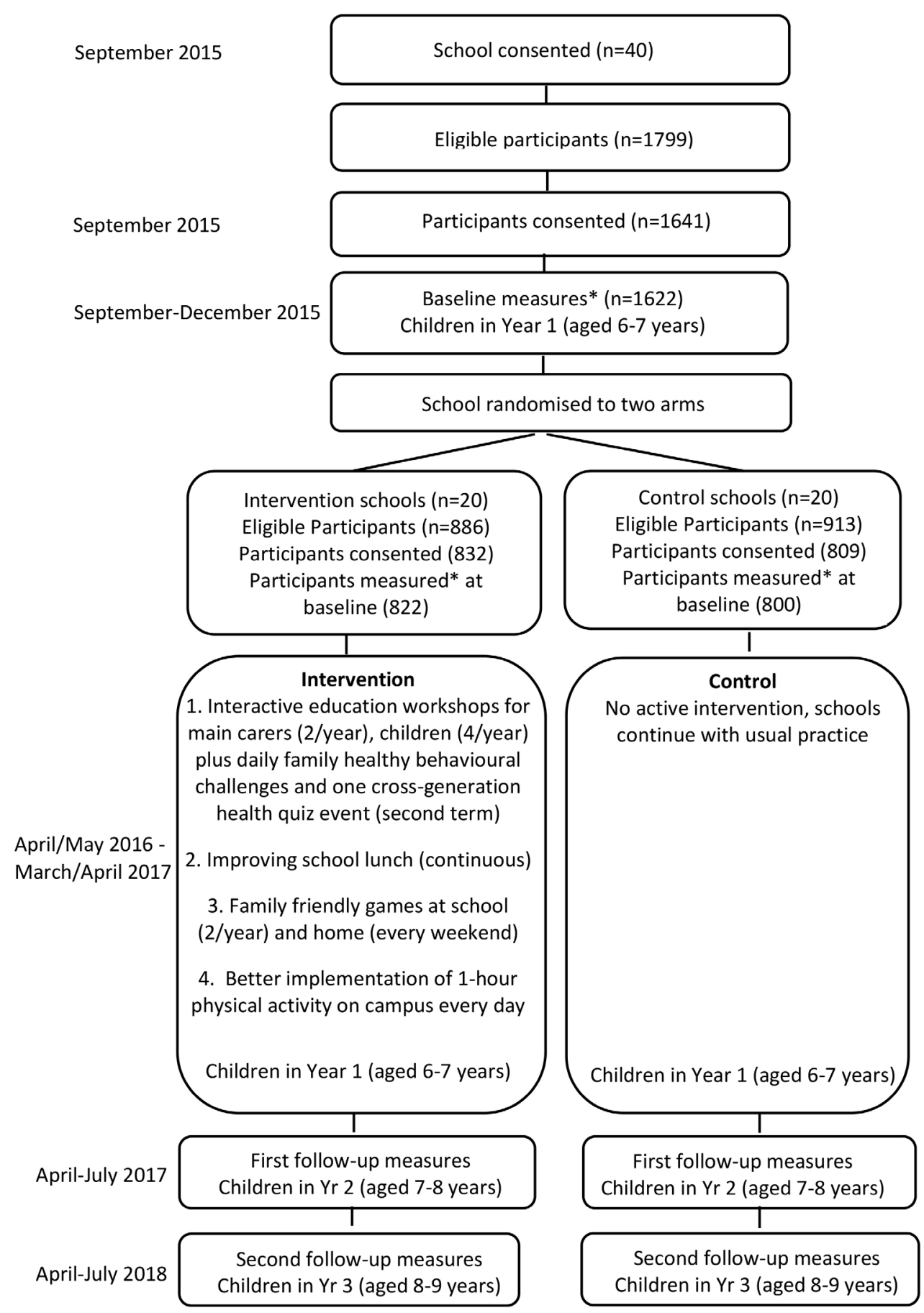

*Children who took part in at least primary outcome (BMI) measures at baseline

Figure 1 Study design and the flow of study participants through the CHIRPY DRAGON study. BMI, body mass index; CHIRPY DRAGON, CHInese pRimary school children PhYsical activity and DietaRy behAviour chanGes InterventiON.

\section{METHODS}

\section{Study setting}

The study will be conducted in the largest Southern Chinese city of Guangzhou, with an urban population of 12.9 million. ${ }^{23}$ Guangzhou, an important centre of China's trade and economic power, has experienced rapid socioeconomic transition in the last three decades.

\section{Study population and participants eligibility}

All year 1 students (average age between 6 and 7 years) from non-boarding, state-funded primary schools in traditional urban districts of Guangzhou are eligible for inclusion. However, a child will be excluded if his or her parents (or guardians) believe he or she should not participate in this study for any medical reasons. 
There are several reasons for targeting this age group. First, there is good evidence of tracking of weight status ${ }^{3}$ and lifestyle ${ }^{24}$ from childhood to later life and adiposity rebound ${ }^{25}$ occurs around the age of 6-7. Furthermore, there are several advantages to targeting primary schools to facilitate or deliver intervention programmes. These include the organisational structure, ${ }^{26}$ and the high level of trust that children and family members attach to primary school staff. ${ }^{16}$ Also, among the key stages of Chinese education for under 18 years-nursery (aged 2-5), primary school (aged 6-12), middle school (aged 13-15) and high school (aged 16-18), the primary school years are the longest and most stable (largely due to the state's compulsory requirement for education starting from primary school). This represents a particular opportunity for delivering and assessing the long-term effects of interventions.

\section{Sampling}

All eligible schools $(n=353)$ will be assigned with a unique identity number and ordered using a random number generator. Schools will be approached sequentially to take part, aiming for 40 schools in total.

\section{School recruitment}

In line with local cultural practice and based on our previous experience of conducting research in Chinese schools, randomly selected schools will be approached through telephone calls and an official letter that shows project approval and support from the local Education and Health Bureaus. The first 40 school principals who agree to participate will be invited to attend a briefing event at the Guangzhou Centre for Disease Control and Prevention (CDC), together with representatives of their district-level education bureaus and CDC.

\section{Method of randomisation and blinding}

Randomisation will take place after consent was taken for the cluster, and after baseline measurements, participating schools will be informed of allocation to ensure allocation concealment.

An independent medical statistician will perform stratified randomisation using the ralloc command in STATA to allocate the 40 schools (clusters) to intervention ( $\mathrm{n}=20$ ) and control $(n=20)$ arms. Four strata will be generated based on two factors which were selected following a discussion with the medical statistician and officials of the local education authority who hold good knowledge of local primary schools. The two factors, considered to be the most important, are (1) whether there is a provision of mid-morning snacks; and (2) whether the school has an indoor activity room. We selected these two factors because they are most likely to impact on the delivery of our intervention programme and ensure a balance across the four strata. We will recruit external workers (independent data collectors who are blinded to study allocation) to undertake all outcome measures.

\section{Participant recruitment}

In Chinese primary schools, there are on average five classes in each year and one class consists of approximately 45 children. For each class, there is a senior teacher (known as class-level head teacher) who is in charge of all children's learning and moral development as well as their general well-being during school hours for the whole 6-year primary school period. Children from all year 1 classes and their family members in the participating schools will be offered the prevention programme. One class will be randomly selected from each participating school to take part in outcome measures. We will ask class head teachers to pass on the study participant information sheet and consent form to parents (or guardians) through the children. Parents (or guardians) of children will also be advised to inform the research team if they believe there are any medical reasons that a child should not participate in any outcome measures and/or intervention activities. Children (and their family members) who return a completed consent form signed by their parents (or guardians) will take part in outcome measures (detailed below). Where we cannot recruit the sufficient number of participants from one class, a second class will be randomly selected.

\section{Trial intervention}

The CHIRPY DRAGON study intervention programme has four components that will be delivered over 12 months by five full-time Chinese staff (known as CHIRPY DRAGON teachers) who are employed and trained by the research team. All intervention components and activities are summarised in table 1 . There are tailored activities for different target groups, including children, parents and grandparents, school staff (principals, physical education (PE) teachers, class teachers) and school lunch providers (menu makers, cooks and other kitchen workers). School principals and class-level head teachers will be provided with a detailed programme handbook which explains the components of the programme and the support required from them. Each of the five CHIRPY DRAGON teachers will be responsible for the facilitation and delivery of the programme activities in four intervention schools. They will also be the first point of contact for any enquires from their respective schools or family members. At the end of each school term, all school principals will be invited to attend a summary meeting at the Guangzhou CDC.

\section{Component 1: interactive learning workshops with coordinated} family-wide healthy behaviour challenges

The overall aim of this component is to improve knowledge of childhood obesity-related behaviours in children and their main carers (predominantly parents and grandparents) through interactive learning activities (run in the school by CHIRPY DRAGON teachers) and to encourage healthy behaviours through family-wide health behaviour challenges (set by children themselves every 2 weeks with guidance from a CHIRPY DRAGON teacher). 
Table 1 Overview of the Chinese primary school children physical activity and dietary behaviour changes intervention programme

\begin{tabular}{|c|c|c|}
\hline Key components & Activities & Target audience \\
\hline \multirow{4}{*}{$\begin{array}{l}\text { Component 1: To improve } \\
\text { childhood obesity-related } \\
\text { knowledge and behaviour } \\
\text { among children and their } \\
\text { main carers }\end{array}$} & (A) Interactive education activities for carers & $\begin{array}{l}\text { Main carers } \\
\text { (parents and } \\
\text { grandparents) }\end{array}$ \\
\hline & (B) Interactive educational activities in children & Children \\
\hline & (C) Family-wide healthy behavioural challenges and child self-monitoring & Children and their \\
\hline & (D) Quizzes for main cares and children & main carers \\
\hline $\begin{array}{l}\text { Component 2: To improve } \\
\text { the nutritional quality of } \\
\text { school lunch provision }\end{array}$ & $\begin{array}{l}\text { (B) Supportive school lunch evaluation and feedback in relation to the } \\
\text { improvement goals }\end{array}$ & \\
\hline $\begin{array}{l}\text { Component 3: To increase } \\
\text { children's physical activity } \\
\text { level outside school }\end{array}$ & (A) Fun and active family games learnt and tried in school & $\begin{array}{l}\text { Children and their } \\
\text { parents }\end{array}$ \\
\hline $\begin{array}{l}\text { Component 4: To increase } \\
\text { children's physical activity } \\
\text { level in school }\end{array}$ & $\begin{array}{l}\text { (B) Setting monthly goals (measurable and achievable) and action plans to } \\
\text { meet, maintain or exceed the national standard and continuous evaluation } \\
\text { and feedback }\end{array}$ & $\begin{array}{l}\text { Children and } \\
\text { school staff }\end{array}$ \\
\hline
\end{tabular}

The interactive learning activities will be delivered in school and tailored to different target audiences, including two workshops for parents and grandparents (if they live with the participating child and/or have important contribution to everyday childcare), four child sessions and two joint child carer/child sessions (quizzes). The key learning messages for the parent/ grandparent workshops will be based on correcting common misperceptions identified through our formative research (eg, belief that children are not susceptible to cardiometabolic complications of obesity or that being overweight is healthy) ${ }^{15}$ as well as about healthy eating, having an active lifestyle and reducing sedentary activity. The sessions will also introduce practical tips for encouraging healthy behavioural change in children. At the end of each session, a colourful educational leaflet which summarises the main learning content will be provided for each participating family to take home. The children's sessions will focus on key messages related to healthy eating (focusing on increasing fruit and vegetables, reducing fat, sugar and salt and appropriate portion size) and an active lifestyle (eg, why being active is important). The joint child carer/child sessions are designed around the key messages delivered in the completed workshops. They will reinforce the messages through cross-generation health quizzes, which aim to motivate, celebrate and award learning.

To coordinate with some of the key health messages learnt at the interactive workshops and to encourage adoption and maintenance of promoted healthy behaviours, CHIRPY DRAGON teachers will ask children to set challenging but achievable individual goals based on their current habits within predetermined themes which will rotate every 2 weeks: eating at least five portions of fruit and vegetables daily; engaging in no more than 2 hours of sedentary screen-based activities a day; consuming less snacks and drinks that are high in sugar and/or fats. In addition, there will be a weekend challenge that aims to promote physical activity involving both the children and their parents (part of component 3, described later). The children can record their achievement with parents' comments on specially designed fun cards which are renewed with each new theme. Every 2 weeks, the responsible CHIRPY DRAGON teacher reviews the cards and provides feedback to those who participate. Children's achievements as well as the involvement of their family members (eg, parental comments given on the fun cards, and attendance at relevant learning workshops) will be recorded on a large CHIRPY DRAGON Reward Board in the format of badges (stamps). There will be one Reward Board for each class. They will be displayed on the central wall of children's classrooms and renewed termly throughout the intervention period. Children who collect the most badges/stamps in a term will be rewarded with a small stationary gift at the end of the joint childcare/ child workshop.

Component 2: setting improvement goals and providing supportive evaluation and feedback for school lunch provision

The overall aim of this component is to improve the nutritional quality of school lunch provision (generally a set lunch box for each child) through (1) introducing five 
school lunch improvement objectives (detailed below) to catering staff who are responsible for producing meals for intervention schools, and (2) regular supportive evaluation and constructive feedback.

The five school lunch improvement objectives were jointly developed by Chinese nutrition experts, obesity prevention specialists within the research team and Chinese school meal providers (including both managers and catering workers). They were tested for feasibility and acceptability in the feasibility trial:

i. Reduce provision of deep fried dishes to no more than once a week.

ii. Reduce portion size of rice (or other starch food such as noodles) in younger children compared with those of older age groups.

iii. Provide vegetables every day (including green leaf vegetables on at least 2 days a week).

iv. Remove processed and high fat meats from the menu.

v. Ensure the amount of cooking salt, added sugars and cooking oil (in each lunch box) does not exceed one-third of the recommended daily intakes for 7-year-old Chinese children. ${ }^{27}$

The objectives allow maximum flexibility for local adoption/adaptation in response to changes in costs, seasonal food availability and local resources.

The implementation of objectives will be achieved through inviting delegates of each catering team (one for each intervention school) to an introductory/training meeting held at the Guangzhou CDC. Regular evaluation of food provision will be undertaken in the form of direct observation (once a month by CHIRPY GRAGON teachers), review of daily ingredient record forms (completed by lead chef) and daily photographs of lunch boxes (submitted weekly by school staff or catering staff). These materials will be reviewed and scored weekly by a member of the research team against the five prespecified school meal improvement objectives (equal weighting for each component, scores ranging 0-5). A written constructive feedback form will be sent to each school and their catering teams every week, highlighting good performance, improvement noticed and/or areas requiring further work. For schools that have achieved a full score for two consecutive weeks within the first month of the intervention period, direct observation and feedback will pause and restart in week 9 . If those schools still meet all improvement objectives in week 9, no more direct observation and feedback will be offered until the start of the next school term. To motivate and recognise good performance or improvements, a recognition certificate will be issued to individual catering teams.

\section{Component 3: promoting physically active games and activities} involving both children and their parents

This component aims to increase children's physical activity level outside school through promoting physical games, exercises or sports activities that involve both children and their parents. To inspire family interest, one session of family friendly games will be delivered to each class (child and one of their parents attend) every school term. To promote maximum practice of activities after the school session, CHIPRY DRAGON teachers will provide taster sessions for two to three games that are easy, fun and safe to play, require little space and no special equipment but allow interaction between children and their parents. Moreover, at the end of each session, CHIRPY DRAGON teachers will assign a family-wide health behaviour challenge to all participating familiesplaying one of the games learnt at school or doing any non-sedentary activities that involve both the child and his/her parents outside school for a minimum of $30 \mathrm{~min}$ each weekend.

Component 4: improving the implementation of the Chinese national requirement for 'One-Hour Physical Activity on Campus Every School Day'

The aim of this component is to increase children's physical activity level in school by improving the implementation of the Chinese national requirement for 'One-Hour Physical Activity On Campus Every School Day' in intervention schools. First, CHIRPY DRAGON teachers will facilitate a staff meeting at each school where school principals, class-level head teachers, PE teachers and student representatives discuss their current situation, identify modifiable barriers to implementation of the national requirement as well as improvement opportunities. The school staff will be encouraged to set measurable action goals and plans for the first month. Schools are encouraged to set achievable goals and plans that suit their own situation. Following the initial meeting, school PE teachers and CHIRPY DRAGON teachers will jointly monitor the implementation of action plans over the month. A follow-up staff meeting will be held where CHIRPY DRAGON teachers provide constructive feedback and action goals and plans will be reviewed and redefined for the subsequent month. For schools that successfully meet all action goals, the goal for the next month would be to maintain the current performance. Monitoring will continue in those schools but no meetings will be held unless there are any setbacks. Monthly meetings will continue for schools that fail to meet their action goals.

\section{Strategies to minimise contamination}

It will be impossible to avoid contamination completely in this trial. However, the risk for contamination will be minimised through a variety of strategies. First, the implementation of component 2 of CHIRPY DRAGON programme involves external catering staff who are likely to serve multiple schools at the same time. Therefore, following randomisation, we checked whether there were any catering companies or teams that prepared lunches for schools from both arms (we found none). We have also asked participating schools to inform us of any changes of school lunch suppliers throughout the whole 
intervention period. Second, we recognise that several social media tools are very popular among the study population and that school teachers often use these tools to communicate with children's parents. Therefore, we will ask staff from the intervention schools not to share any information about the intervention programme through social media. We will also ask school staff not to share any intervention information with the media. Most intervention components could only be implemented through school and unlikely to be transferrable to families in other schools. Furthermore, following randomisation, the distance between control and intervention schools was checked and no instances were found where an intervention and control school were co-located in the same natural community.

\section{Comparator}

No active intervention will be provided to schools in the control arm. They will continue with any ongoing health-related activities.

\section{Outcome measures}

The primary outcome will be BMI z-score (based on WHO 2007 Growth Charts). ${ }^{28}$ Secondary outcomes are: (1) anthropometric measures (including percentage of overweight and obesity as defined by WHO 2007 Growth Charts, waist circumference and body fatness); (2) dietary behaviour (including percentage of children consuming $\geq 5$ portions of fruit and vegetables daily, daily average servings of fruit and vegetables and weekly average servings of unhealthy snacks and sugar-added drinks); (3) physical activity (including time in moderate to vigorous physical activity (MVPA) (minutes/24hours), percentage of children achieving $\geq 60$ min MVPA in 24 hours and percentage of children engaging in active sports, dance or games for at least once in the weekend) and sedentary behaviour (including sedentary time (minutes/24hours); screen viewing behaviour); (4) psychosocial outcomes to assess the wider effects of the intervention, including benefits and potential harms (health-related quality of life and bullying). Measures will be undertaken at baseline, immediately following the intervention period and 12 months after the intervention period is completed.

\section{Data collection methods}

Assessments will be undertaken in school by independent trained research staff who are blinded to school allocation using standardised procedures and validated or adapted instruments at baseline and follow-up time points. In addition, parents and other live-in adult family members of participating children will be asked to complete questionnaires at each time point. These cover questions on the dietary, sedentary, physical and sleep activity habits of the child and the family, built neighbourhood environment, quality of life, perception of child weight status, child care arrangement and household expenditure. Date of birth, sex and child birth weight and other basic demographic data of participating children will be obtained from the parent questionnaire. Children will also be invited to complete a 24-hour diary (on health-related behaviour) as well as a researcher-administered questionnaire (on family lifestyle and psychosocial measures). A summary of key measurements undertaken in this study is included in online supplementary file 1 .

\section{Anthropometric measures}

All measurements will be undertaken barefoot and in light clothing. Standing height will be measured at least twice with a TGZ-type height tester (Dalian); a third measure will be taken if the difference between the first two is larger than $0.4 \mathrm{~cm}$. Weight will be measured with an electronic scale (JH-1993T, weighing Apparatus Co. Ltd., Dalian, Dalian, China). Body fat percentage will be measured using a single-frequency ImpediMed machine (ImpDF50; Impedimed, Pinkenba, Australia). Waist circumference (measured midway between the rib cage and the iliac crest) will be measured at least twice (a third measure will be taken if the difference between the first two is bigger than $0.4 \mathrm{~cm}$ ) using a non-stretch tape measure.

\section{Health behaviour measures}

Child dietary assessment methods include a parent questionnaire and a child diary, which include validated (University of Leeds short form food frequency questionnaire (SFFQ); Day in Life Questionnaire ${ }^{29}$ and purposely developed items.

To measure child physical activity and sedentary behaviours, we will use purposely developed questions and adapt validated (Godin Leisure-Time Exercise Questionnaire $^{30}$; Day in Life Questionnaire ${ }^{29}$ and Physical Activity Questionnaire for Children (PAQ-C) $)^{31}$ tools, and administer these through the parent questionnaire, child questionnaire and child diary. We will also use a validated objective method (GENEActiv Original, Activinsights, Cambridge, UK) ${ }^{32}$ to assess physical activity and sedentary behaviours of the children. All children taking part in outcome measures will be invited to wear the wrist-worn accelerometer continuously for 5 days (including three weekdays and a weekend). MVPA (minutes/24hours) and sedentary time (minutes/24hours) will be derived and analysed using the methods described by Noonan and colleagues. ${ }^{33}$

\section{Blood pressure}

Blood pressure will be measured using clinically validated, automated monitors (Omron HEM-7211, Dalian). We will use the appropriate cuff size for each child. Following a seated rest of $3 \mathrm{~min}$, two readings will be taken with a 3 min rest interval. If an error reading occurs or one of the values is outside the normal range, a third measurement will be taken.

\section{Other measures}

Quality of life in children will be measured using the validated Chinese version of Pediatric Quality of Life Inventory TM (PedsQL 4.0) ${ }^{34}$ and The Child Health Utility 
9D (CHU 9D).$^{35}$ These questions will be administered to children on a one-to-one basis by trained researchers. In addition, standardised questions (derived either from validated questionnaires or purposely developed) will be asked to understand the child's perceptions of family and peer support for physical activity, parental sedentary behaviour as well as their perceptions of the built and social neighbourhood environments. ${ }^{36}$ In addition, a translated version of the social acceptance domain from the KIDSCREEN-52 Health Questionnaire for Children and Young People will be included. ${ }^{37}$ As part of the questionnaires for parents and other live-in adult family members, we will use the validated Chinese version of EuroQol-5D (EQ-5D) ${ }^{38}$ to measure their quality of life.

\section{School-level data}

To allow exploration and analysis of potential moderators of observed intervention outcomes beyond the level of the child and their family, several school characteristics will be measured. Information such as the provision of and participation in school breakfast, mid-morning snack and lunch, measured size of school playgrounds and the availability of an indoor activity room will be collected. In addition, as part of process evaluation (described below) and to allow exploration and analysis of potential mediators of observed intervention outcomes, the individual school's response (in terms of participation and progress) to lunch intervention (component 2) and campusbased physical activity intervention (component 4 ) will be recorded.

\section{Process evaluation}

In line with the process evaluation guidance set out by the UK MRC, ${ }^{39}$ a range of methods will be used to evaluate intervention implementation, mechanisms of impact and contextual factors. These include (1) activity implementation records (standardised forms to record key information relating to adoption, dose, reach for each programme component), (2) regular direct observation by trained individuals who are not involved in the delivery of the programme (standardised questionnaire to aid evaluation of all aspects of implementation fidelity), (3) minutes of implementation team meetings (with a focus on understanding, handling and recording necessary adaptations and unexpected adverse events), (4) qualitative evaluation (understanding participant experience and responses through personal interviews with school principals and focus groups with school teachers, family members and children), (5) a summary of individual school's cooperation level (describing school leaders and teachers' working styles, attitudes toward the programme and their level of cooperation) and (6) records on the individual participant's participation and behavioural responses to intervention activities. Both school-level and individual-level data on intervention participation and behavioural responses can be used in moderator and/or mediator analyses to explore mechanisms of impact.

\section{Intervention logic model}

The intervention components and impact pathways are summarised in a logic model (figure 2).

\section{Sample size calculation}

Statistical considerations

The primary objective of the study is to compare the difference in mean BMI z-score between the intervention and control arms at the end of the intervention (at the first follow-up). For the sample size calculation, we considered the following available information. Our cross-sectional study in the same setting ${ }^{19}$ found that the SD of BMI z-score was 1.39 with intraclass correlation coefficient of $<0.01$. Clustering will be allowed for at the level of the school (in this study, one cluster refers to one class because in each participating school, we will aim to randomly select one class to take part in outcome measures). In cases where multiple classes will be included in each school, we will include both levels of clustering where possible. A change of 0.25 z-scores has been shown to be associated with clinically detectable benefits in obese adolescents. ${ }^{40}$ However, even much smaller reductions in BMI z-score (0.1 units) may have clinically important effects in this age group. ${ }^{41}$ Correlation between baseline and follow-up BMI z-score was found to be 0.70 in a previous trial of obesity prevention conducted by members of our research team among South Asian primary school children. ${ }^{42}$ A review of childhood obesity intervention studies in China found that in 10/22 included studies, there were no dropouts, and for the remainder, exact dropout rates were not reported. ${ }^{43}$ Based on anecdotal research experience in this study setting, we estimated conservatively a dropout rate of $10 \%$ for this trial. The above information allowed us to define sample size for this trial.

\section{Defining sample size}

Using the average class size of 45 in Guangzhou and assuming a $10 \%$ dropout rate by the end of the study, with correlation between baseline and follow-up being 0.70 and a conservative ICC being 0.01 , a sample size of 1640 recruited from 40 schools (20 intervention) is sufficient to detect a difference of 0.17 units (midpoint for estimates of minimal clinically important differences) in the mean BMI z-score with a power of $80 \%$ and a significance level of $5 \%$. This detectable difference was estimated using the STATA function clustersampsi. ${ }^{44}$

\section{Planned statistical analysis}

All analyses will be by intention to treat. The baseline characteristics for participating pupils will be described by control and intervention groups. The differences in primary and other outcomes between the intervention and control groups will be examined at both follow-up time points (immediately following the intervention, and 12 months postintervention), with the first follow-up analysis being the primary evaluation of intervention effects. As randomisation will be at the school (cluster) level, appropriate statistical methods to 


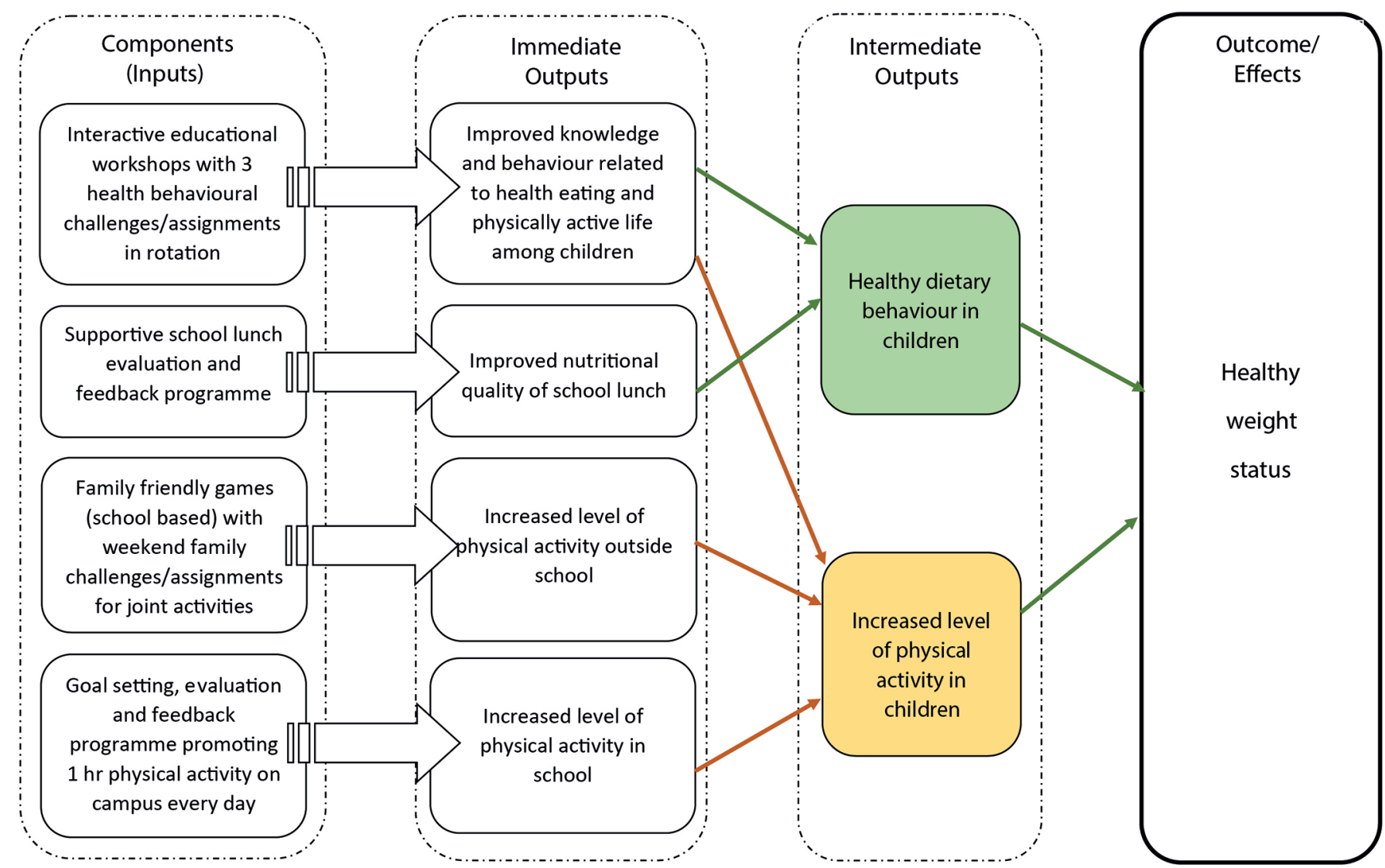

Figure 2 Logic model illustration. The theoretical pathway leading from the intervention to improved health outcome among the programme participants.

account for the clustering within schools will be used in the analysis.

The primary aim of the study is to evaluate whether the change in BMI z-score differs between arms. In statistical terms, this null hypothesis (no difference) can be tested using a mixed linear model with follow-up BMI z-scores as the dependent variable and baseline BMI z-scores and treatment arm as the independent variables. These models will be fitted using mixed models in STATA.

Secondary outcomes are either binary (eg, engaging in at least $60 \mathrm{~min}$ of moderate to vigorous physical activity a day or not) or continuous (eg, daily minutes of sedentary behaviour), and therefore either logistic or linear link functions will be used, with transformations where appropriate to accommodate any non-normality. All model assumptions will be checked, goodness of fit explored. The primary analysis will be adjusted for clustering and baseline value of the outcome only. Secondary analyses will additionally adjust for prespecified school-level and child-level covariates. These include those that were used in randomisation (ie, whether the school provides mid-morning snack, whether the school has an indoor activity room) and important sociodemographic (ie, sex and parental education level) and health behaviour factors (fruit and vegetables, unhealthy snacks and sugaradded drink consumption and minutes/day MVPA and sedentary time). Where appropriate, multiple imputation methods will be used to account for missing data.

All outcome and all subgroup comparisons at both time points will be considered significant at the $5 \%$ level (and so $95 \%$ CIs reported). Treatment effects will be reported on the difference scale (ie, mean difference).

\section{Planned subgroup analyses}

We will also examine whether any difference in outcomes between control and intervention arms varies by sex, socioeconomic factor (mother's education level will be used, categorised as did or did not attend university) and weight status at baseline (overweight/obese as compared with non-overweight/obese).

\section{Economic evaluation}

The economic evaluation will be conducted alongside the trial to estimate the difference in costs and outcomes between the situation of supplying the intervention package and a situation where no intervention is in existence (ie, usual current practice). Both a cost-utility analysis (CUA) and a cost-effectiveness analysis (CEA) will be conducted. The primary measure of effectiveness for the CEA will be change in BMI z-score between the arms. The primary outcome measure for the CUA will be quality-adjusted life year (QALY) calculated from data collected using the CHU9D instrument, a paediatric utility-based 
quality of life measure validated in a Chinese population. ${ }^{35}$ Costs collected will focus on costs that are likely to differ between the intervention and control arm: set up and delivery of intervention; staff costs; materials used during sessions; and impact of intervention on household food expenditure. The trial facilitators will supply all data on intervention-related costs. Costs linked to household expenditure will be collected from participants by asking them to complete a parent report. Unit costs will be obtained from Chinese sources, or valued at market prices. The costs will be estimated by calculating the resource use multiplied by the unit cost. Total costs for the intervention as well as average cost per participant will be estimated. Using regression analysis, we will control for differences in characteristics of participants and baseline CHU9D scores.

The economic evaluation will be conducted from both a public sector and societal perspective. The public sector perspective will only include costs linked to delivery of the intervention alongside the average QALY impact on the children; the societal perspective will broaden the framework to include household expenditure and intervention effect on parents and other household adult members. To facilitate this broader perspective, QALYs for parents and other adult household members will be estimated using data collected from the EQ-5D instrument. Costs and outcomes will be combined using incremental cost-effectiveness ratio (ICER) and expressed as cost per QALY gained. This information can then be used by decision-makers to judge the cost-effectiveness. As no equivalent threshold value exists within the Chinese setting for how much decision-makers are willing to pay for a unit gain in QALY, the ICER will be assessed with reference to the established UK and US threshold values.

Uncertainty in the data will be assessed using non-parametric bootstrapping and decision uncertainty will be represented using cost-effectiveness acceptability curves.

The within-trial analysis will only use data from the trial, and if the intervention demonstrates effectiveness within that time period, we will develop a decision-analytic model to estimate the cost-effectiveness beyond the trial period. This will project costs and outcomes over a lifetime and probability sensitivity analysis will be applied to reflect the uncertainty within the model, and the data used to populate the model.

\section{Trial status}

Recruitment of schools and participantsstarted in September 2015 and completed by the end of September 2015. Baseline measures for the first 20 schools (10 in each arm) commenced in September 2015 and completed in October 2015. For the last 20 schools (10 in each arm), baseline measures started in November 2015 and completed in December 2015. The 12-month intervention programme started in March/April 2016 for the first 10 intervention schools and in April/May for the remaining 10 intervention schools.

\section{Ethics and dissemination}

Ethical approval was obtained from the Life and Health Sciences Ethical Review Committee at the University of Birmingham (ERN_14-1440). Local ethical approval was also granted by the Ethical Committee of Guangzhou Centre for Disease Control and Prevention. The primary, secondary, process evaluation and economic evaluation results of the trial will be disseminated through relevant international peer-reviewed journals and conferences. On completion of the trial, and after publication of these results, data would be available on request by contacting the first author of this protocol.

\section{DISCUSSION}

The CHIRPY DRAGON study is a cluster-randomised controlled trial which aims to evaluate the clinical and cost-effectiveness of an obesity prevention programme for urban Chinese primary school-aged children. The intervention programme is school based with family-wide engagement and activities, and focuses on promoting healthy behaviours in children through four coordinated components: improving health knowledge, skills and behaviour among children and their primary carers in the family (component 1 ); improving the nutritional quality of school lunch (component 2); increasing physical activity level outside school (component 3 ) and increasing physical activity level on campus (component 4).

To our knowledge, CHIRPYDRAGON is the first childhood obesity prevention intervention in China that is developed rigorously using mixed methods following the framework set out by the UK MRC. We have an explicit process evaluation plan that is designed following the guidance recently published. This includes data collection on immediate and intermediate behavioural outputs, at both school and individual levels. Data of this type have rarely been collected and examined in previous trials but is essential for understanding implementation context and for the analyses of potential moderation/mediation effects. Other strengths of the study include incorporating behavioural change techniques in the design of the intervention programme; a sample size that is likely to be large enough to detect clinically significant differences in the primary outcome and offers the possibility for exploring effects in subgroups; and use of a validated and objective measure of physical activity. Finally, the study is built on a strong partnership between the research team at the University of Birmingham and Chinese local health and educational authorities whose mission is to promote good health and learning in school children. This will allow a faster track to knowledge transfer and dissemination of research impact through the rolling out of the intervention programme, if it is found to be cost-effective.

While the study includes a large number of children, the number of clusters is still relatively small. As a result, cluster-level baseline imbalance may be present despite randomisation. Although we plan to adjust for important covariates in secondary analyses, it is possible that not all important potential confounders will be measured and 
included. Moreover, as with any school-based childhood obesity prevention trial, contamination between the two arms cannot be avoided completely. However, we anticipate the risk for contamination is small and we have set out specific strategies to minimise such a risk.

Acknowledgements We sincerely thank the funder, Chinese local authorities, schools, parents and children who participate in or support this study.

Contributors All authors contributed to the development of the design of the CHIRPY DRAGON Trial. Intervention development was led by BL, with advice from $\mathrm{PA}$ and contributions from MP, KKC, KH, EF and WJL. BL, PA, MP, EF and KH form the project management group and advised on the trial design, measurement tools and project overview. KH advised on the sampling, sample size estimation and the statistical analysis plan. JM performed randomisation with support from $\mathrm{KH}$. $\mathrm{EF}$ designed the economic evaluation. BL designed process evaluation tools with advice from PA and MP, BL oversees and manages the project fieldwork with WJL being the local supervisor. WJL, RL and WL contribute to the implementation of the project in Guangzhou. Funding for the trial was secured by BL, with support from PA and KKC. BL wrote the first draft of the manuscript. All authors contributed to critical revisions of the paper. The final manuscript was read and approved by all authors.

Funding Following external scientific review and approvals from the Chinese authority, the University of Birmingham received a charitable donation from Zhejiang Yong Ning Pharmaceutical Ltd Co. to support its research collaboration with China Guangzhou Centre for Diseases Control and Prevention in obesity prevention among Chinese children. The research is led and hosted by the University of Birmingham.

Disclaimer The funder has no role in any aspects of the study's design, implementation, analyses and reporting.

Competing interests None declared.

Provenance and peer review Not commissioned; externally peer reviewed.

Open Access This is an Open Access article distributed in accordance with the Creative Commons Attribution Non Commercial (CC BY-NC 4.0) license, which permits others to distribute, remix, adapt, build upon this work non-commercially, and license their derivative works on different terms, provided the original work is properly cited and the use is non-commercial. See: http://creativecommons.org/ licenses/by-nc/4.0/

(c) Article author(s) (or their employer(s) unless otherwise stated in the text of the article) 2017. All rights reserved. No commercial use is permitted unless otherwise expressly granted.

\section{REFERENCES}

1. International Food Policy Research Institute. Global Nutrition Report 2016: From Promise to Impact: Ending Malnutrition by 2030. Washington: International Food Policy Research Institute, 2016.

2. Lobstein T, Baur L, Uauy R. Obesity in children and young people: a crisis in public health. Obes Rev 2004;5 Suppl 1:4-85.

3. Singh AS, Mulder C, Twisk JW, et al. Tracking of childhood overweight into adulthood: a systematic review of the literature. Obes Rev 2008;9:474-88.

4. Baker JL, Olsen LW, Sørensen TI. Childhood body-mass index and the risk of coronary heart disease in adulthood. N Engl J Med 2007:357:2329-37.

5. Wardle J, Brodersen NH, Cole TJ, et al. Development of adiposity in adolescence: five year longitudinal study of an ethnically and socioeconomically diverse sample of young people in Britain. BMJ 2006;332:1130-5.

6. Serdula MK, Ivery D, Coates RJ, et al. Do obese children become obese adults? a review of the literature. Prev Med 1993;22:167-77.

7. Ji CY, Chen TJ. Empirical changes in the prevalence of overweight and obesity among Chinese students from 1985 to 2010 and corresponding preventive strategies. Biomed Environ Sci 2013;26:1-12.

8. Wang Y, Lobstein T. Worldwide trends in childhood overweight and obesity. Int J Pediatr Obes 2006;1:11-25.

9. Ji CY, Cheng TO. Prevalence and geographic distribution of childhood obesity in China in 2005. Int J Cardiol 2008;131:1-8.

10. Ji CY. Report on childhood obesity in China (4) prevalence and trends of overweight and obesity in Chinese urban school-age children and adolescents, 1985-2000. Biomed Environ Sci 2007;20:1-10.
11. Waters E, de Silva-Sanigorski A, Hall BJ, et al. Interventions for preventing obesity in children. Cochrane Database Syst Rev 2011:CD001871.

12. Medical Research Council. A framework for development and evaluation of RCTs for complex intervnetions to improve health. London: Medical Research Council, 2000.

13. Campbell NC, Murray E, Darbyshire J, et al. Designing and evaluating complex interventions to improve health care. BMJ 2007;334:455-9.

14. Li B, Liu WJ, Cheng KK, et al. Development of the theory-based Chinese primary school children physical activity and dietary behaviour changes intervention (CHIRPY DRAGON): development of a cluster-randomised controlled trial. The Lancet 2016;388:S51.

15. Li B, Adab P, Cheng KK. The role of grandparents in childhood obesity in China - evidence from a mixed methods study. Int $J$ Behav Nutr Phys Act 2015;12:91.

16. Li B. Childhood Obesity Prevention in China: A Mixed-Methods Approach to Inform Development of Theoretically Based Interventions.PhD thesis, University of Birmingham 2013.

17. Li B, Adab P, Cheng KK. Stakeholder views on why childhood obesity is rising in China: a qualitative study. 12th International Congress on Obesity, Malaysia. Obesity Reviews 2014;15:203.

18. Li B, Adab P, Cheng KK. What and how childhood obesity prevention interventions should be delivered in China: a qualitative study. The 12th International congress on obesity obesity review. 2014;15:237.

19. Li B, Adab P, Cheng KK. Family and neighborhood correlates of overweight and obesogenic behaviors among Chinese children. Int $J$ Behav Med 2014;21:700-9.

20. Li B, Adab P, Keung Cheng K, et al. Systematic review of modifiable risk factors associated with childhood obesity in China, a population undergoing rapid social and economic transition. Appetite 2015;87:376.

21. Kotler P, Roberto N. Lee Nancy: Social Marketing - Improving the Quality of Life. London: Sage Publications, 2002.

22. Michie S, Ashford S, Sniehotta FF, et al. A refined taxonomy of behaviour change techniques to help people change their physical activity and healthy eating behaviours: the CALO-RE taxonomy. Psychol Health 2011;26:1479-98.

23. Lu SM, Zhu Y. Residential mobility within guangzhou City, China, 1990-2010: local residents versus migrants. Eurasian Geogr Econ 2014:55:313-32.

24. Wang Y, Bentley ME, Zhai F, et al. Tracking of dietary intake patterns of Chinese from childhood to adolescence over a six-year follow-up period. J Nutr 2002:132:430-8.

25. Rolland-Cachera MF, Deheeger M, Bellisle F, et al. Adiposity rebound in children: a simple indicator for predicting obesity. Am J Clin Nutr 1984;39:129-35.

26. Story M, Kaphingst KM, French S. The role of schools in obesity prevention. Future Child 2006;16:109-42.

27. The Chinese Nutrition Association. Chinese Dietary Reference Intakes Handbook. Beijing: Chinese Standard Publishing, 2013.

28. Word Health Organization. The WHO child growth standards, 2007.

29. Roberts KFS. Review of dietary assessment methods in public health Oxford. National Obesity ObservatoryNational Obesity Observatory: Oxford, 2010.

30. Godin G, Shephard RJ. Godin leisure-time exercise questionnaire. Med Sci Sports Exerc 1997:S36-S38.

31. Kowalski KC, Crocker PR, Donen RM. The physical activity questionnaire for older children (paq-c) and adolescents (paq-a) manual: College of Kinesiology, University of Saskatchewan, 2004.

32. Phillips LR, Parfitt G, Rowlands AV. Calibration of the GENEA accelerometer for assessment of physical activity intensity in children. J Sci Med Sport 2013;16:124-8.

33. Noonan RJ, Boddy LM, Kim Y, et al. Comparison of children's freeliving physical activity derived from wrist and hip raw accelerations during the segmented week. J Sports Sci 2017;35:2067-72.

34. Chen YM, He LP, Mai JC, et al. Nalidity and reliability of pediatric quality of life inventory version 4.0 Generic core scales in Chinese children and adolescents]. Zhonghua Liu Xing Bing Xue Za Zhi 2008:29:560-3.(Published In Chinese language).

35. Stevens KJ. Working with children to develop dimensions for a preference-based, generic, pediatric, health-related quality-of-life measure. Qual Health Res 2010;20:340-51.

36. Huang YJ, Wong SH, Salmon J, et al. Reliability and validity of psychosocial and environmental correlates measures of physical activity and screen-based behaviors among Chinese children in Hong Kong. Int J Behav Nutr Phys Act 2011;8:16.

37. Ravens-Sieberer U, Gosch A, Rajmil L, et al. The KIDSCREEN-52 quality of life measure for children and adolescents: psychometric results from a cross-cultural survey in 13 European countries. Value Health 2008;11:645-58. 
38. Wu C, Gong Y, Wu J, et al. Chinese version of the EQ-5D preference weights: applicability in a Chinese general population. PLoS One 2016;11:e0164334.

39. Moore GF, Audrey S, Barker M, et al. Process evaluation of complex interventions: Medical Research Council guidance. BMJ 2015;350:h1258.

40. Ford AL, Hunt LP, Cooper A, et al. What reduction in BMI SDS is required in obese adolescents to improve body composition and cardiometabolic health? Arch Dis Child 2010;95:256-61.

41. Kolsgaard ML, Joner G, Brunborg C, et al. Reduction in BMI z-score and improvement in cardiometabolic risk factors in obese children and adolescents. the oslo adiposity intervention study - a hospital/ public health nurse combined treatment. BMC Pediatr 2011;11:47.

42. Adab P, Pallan MJ, Cade J, et al. Preventing childhood obesity, phase II feasibility study focusing on South Asians: BEACHeS. BMJ Open 2014;4:e004579.

43. Li M, Li S, Baur LA, et al. A systematic review of school-based intervention studies for the prevention or reduction of excess weight among Chinese children and adolescents. Obes Rev 2008;9:548-59.

44. Hemming K. Marsh J. A menu-driven facility for sample-size calculations in cluster randomized controlled trials. Stata Journal 2013;13:114-35. 